\title{
The Utility of Fibrinogen/C-reactive protein Ratio versus D-dimer and Fibrin Degradation Product in Diagnosis of Overt Disseminated Intravascular Coagulation in Intensive Care Unit patients
}

\author{
Sherif W. Nashed, Ahmed A. Elshebieny, Mohammed M. Maarouf, Nancy R. Nawar \\ Department of Anesthesiology, Faculty of Medicine - Ain Shams University \\ Corresponding author: Nancy R. Nawar; Mobile: 01001023246; Email: nancy_raouf1@ hotmail.com
}

\begin{abstract}
Background: Coagulopathies in ICU are one of the most common complications in ICU. DIC is one of the most threatening coagulopathies in ICU.It is accompanied with high mortality rates in ICU.Early diagnosis of DIC is very important for early intervention for better prognosis.DIC is caused by several pathologies like sepsis, trauma, obstetric causes and malignancies. Aim of the Work: to compare between the sensitivity between fibrinogen/CRP ratio and FDP and D-dimer. Patients and Methods: After obtaining approval from the medical ethical committee at Ain Shams University, this observational prospective study was conducted at Ain Shams University Intensive Care Units. The study included 70 patients in Demerdash ICU after including patients with ISTH score $\geq 5$. 2 samples were collected from each patient. Results: The results were in favor to the classical parameters of diagnosis of overt DIC in comparison with the new parameter of DIC. It showed that D-dimer and FDPs together were more sensitive than fibrinogen/CRP ratio. Conclusion: The D-dimer and FDP tests offered the best test panel in the diagnosis of DIC especially in overt DIC due to their high sensitivity.
\end{abstract}

Key words: fibrinogen, C-reactive protein, D-dimer, fibrin degradation product, overt disseminated intravascular coagulation, ICU

\section{INTRODUCTION}

Disseminated intravascular coagulation (DIC) is a serious, life-threatening condition in which the proteins in the blood involved in blood clotting become overactive ${ }^{(1)}$.

The subcommittee on DIC of the International Society on Thrombosis and Haemostasis has suggested the following definition for DIC: "An acquired syndrome characterized by the intravascular activation of coagulation with loss of localization arising from different causes. It can originate from and cause damage to the microvasculature, which if sufficiently severe, can produce organ dysfunction" (2).

DIC is most commonly observed in severe sepsis and septic shock. Indeed, the development and severity of DIC correlate with mortality in severe sepsis ${ }^{(3)}$.

The symptoms of DIC are often those of the underlying inciting condition. In addition, symptoms of thrombosis, embolism, organ dysfunction, or bleeding may be present ${ }^{(4)}$.

\section{AIM OF THE WORK}

The aim of this work was to compare between the sensitivity of fibrinogen/CRP ratio and FDP and D-dimer.

\section{PATIENTS AND METHODS}

Study setting:After obtaining approval from the medical ethical committee at Ain Shams University, this study was conducted at Ain Shams University Intensive Care Units.
Type of Study: Observational Prospective study.

Study Period: 3 months.

\section{Study Population}

Inclusion Criteria: Adult patients aging between 20-70 years in both sexes. General surgery and obstetric patients. American society of anesthesiologists ASA III and ASA IV.

Exclusion Criteria: ASA V. Patients whom were presented with isolated thrombosis. 3) Hepatic patients with thrombocytopenia. Patients below and above the desired age group. Patients presented with chronic DIC.

Sampling Method: An observational prospective study was found to be the most suitable design in order to achieve the study objectives.

Sample Size: Using PASS program, setting alpha error at $5 \%$ and power at $80 \%$. Result from previous study Kimet al. ${ }^{(5)}$ showed that the sensitivity of Fibrinogen/CRP ratio for detecting DIC cases was $67 \%$ compared to $91 \%$ as reported by Yu et al. ${ }^{(6)}$ for D-dimer and FDPs. Based on this conclusion, , the needed sample was 70 DIC cases.

Study Procedures: The International Society for Thrombosis and Haemostasis (ISTH) has adopted a score that assists in the diagnosis and the identification of patients at risk for the development of DIC. The score is based on readily available coagulation assays including Prothrombin time PT, Platelets count, fibrinogen and Ddimer ${ }^{(7)}$.Patients who fulfilled terms of ISTH score 
as shown in the following table were admitted to the study.

After written of consent, a peripheral blood sample was withdrawn from suspected overt DIC patients according to ISTH scoring system by using the following terms:

\begin{tabular}{|l|}
\hline Platelet count: \\
\hline$>10010^{\wedge} 3 /$ microL $\ldots \ldots \ldots \ldots$. \\
\hline $50-10010^{\wedge} 3 /$ microL $\ldots \ldots \ldots \ldots \ldots 1$ \\
\hline$<5010^{\wedge} 3 /$ microL $\ldots \ldots \ldots \ldots \ldots .2$ \\
\hline PT: \\
\hline$<3 \mathrm{sec} \ldots \ldots \ldots \ldots . \ldots$ \\
\hline$>3 \mathrm{sec}-<6 \ldots \ldots \ldots .1$ \\
\hline$>6 \mathrm{sec} \ldots \ldots \ldots \ldots .2$ \\
\hline Fibrinogen: \\
\hline$>1 \mathrm{~g} / \mathrm{L}=>100 \mathrm{mg} / \mathrm{dl} \ldots 0$ \\
\hline$<1 \mathrm{~g} / \mathrm{L} \ldots \ldots \ldots \ldots \ldots 1$ \\
\hline D-Dimer normal range $<300$ \\
\hline No increase $\ldots \ldots \ldots \ldots . .0$ \\
\hline Moderate increase $\ldots \ldots .2$ \\
\hline Marked increase $\ldots \ldots \ldots .3$ \\
\hline
\end{tabular}

Patients with score $>5$ were included in the study.

Data collection CRP, Fibrinogen, D-dimer and Fibrin degrading products.

\section{Study Intervention}

Study groups: Two samples were collected from each patient with DIC and the samples were divided into 2 groups and each group will include 1 of the two samples of each patient: Study group\#1: Fibrinogen and CRP were data collected and ratio was calculated. Study group\#2:

D-dimer and FDPswere data collected

\section{Measurements}

Age limit: A range of age limit in between 20 to 30 (mainly obstetric patients), 30 to 40 (mainly postoperative and trauma patients), 40 to 50(mainly postoperative patients) and 50 to 60 (mainly malignancies and postoperative patients)

American Society of Anesthesiologist (ASA score) classification ${ }^{(7):}$ Patients were mainly ASA III and ASA IV.

Etiology of DIC: Postsurgical, obstetrics, sepsis, polytrauma and malignancies.

\section{Fibrinogen/C-reactive protein ratio}

Fibrinogen were sampled from each of 70 patients and the CRP as well and both results were data collected and positive results were organized and tabulated.

\section{D-dimer and Fibrin degradation products}

Also, data collected were from all 70 patients also and positive results were organized.

\section{Comparison between the two results.}

\section{The plan}

70 cases of patients whom were proven to be suffering from DIC according to ISTH score.

Each case had blood venous samples for CRP, Fibrinogen, FDPs and D-dimer.

And then the samples were collected as two groups

First group: 70 samples including positive samples for Fibrinogen/CRP ratio

Second group: 70 samples including positive samples for FDPs (fibrin degradation product) and Ddimer.

\section{The samples were taken as:}

CRP(C-reactive protein) was sampled by turbidmetry technique (latex) which is more sensitive technique by a red capped tube by Heels company kit, this sample was done in serology laboratory in the main laboratory in internal medicine hospital Ain Shams hospital.

D-dimer was sampled in blue capped tube by D-dimer inovance kit in CA 1500 Sysmex machine in hematology labs for coagulation and hemmorage in Clinical pathology hematology department.

Fibrinogen was sampled in blue capped tubes by Multifibrin U kits by using CA 1500 Sysmex machine in hematology labs for coagulation and hemorrhage in Clinical pathology hematology department.

Fibrin Degradation Products were sampled in blue capped tubes using latex manual technique by using El Sharkia company Kits in hematology labs for coagulation and hemorrhage in Clinical pathology hematology department.

The patients with Fibrinogen/CRP ratio $<104$ were considered positive value.

The patients with D-dimer $>0.50 \mu \mathrm{g} / \mathrm{mg}$ with FDPs $>40 \mathrm{mg} / \mathrm{dl}$ were considered positive value.

All results were collected and tabulated.

\section{Statistical Analysis}

Statistical analysis was performed using Microsoft Excel version 2010 and Statistical Package for Social Sciences (SPSS) for Windows version 20.0. 
Data was described as range, mean and standard deviation (for numeric parametric variables), range, median and interquartile range (for numeric nonparametric variables), or number and percentage (for categorical variables). Difference between two groups were analyzed using independent student's t-test (for numeric parametric variables), Mann-Whitney's U-test (for numeric non-parametric variables), or Chi-squared test (for categorical variables). Receiver operator characteristics (ROC) curves were instructed for measured variables (fibrinogen, CRP and their ratio) as predictors of overt DIC and accuracy were expressed in terms of sensitivity, specificity, positive and negative predictive values. Significance level was set at 0.05 .

Probability (P-value): P-value $<0.05$ was considered significant. P-value $<0.001$ was considered as highly significant. $\mathrm{P}$-value $>0.05$ was considered insignificant.

\section{RESULTS}

Table (1): Age distribution of the study group ( $\mathrm{N}=70)$.

\begin{tabular}{|l|c|c|}
\hline \multicolumn{1}{|c|}{ Age (years) } & No. & \% \\
\hline $20-30 y$ & 21 & $30.0 \%$ \\
\hline$>30-40 \mathrm{y}$ & 24 & $34.3 \%$ \\
\hline$>40-50 \mathrm{y}$ & 19 & $27.1 \%$ \\
\hline $50-60 \mathrm{y}$ & 5 & $7.1 \%$ \\
\hline$>60 \mathrm{y}$ & 1 & $1.4 \%$ \\
\hline Total & 70 & $100.0 \%$ \\
\hline Range $[$ Mean \pm SD] & $20-65[36.52 \pm 11.81]$ \\
\hline
\end{tabular}

This table shows that the ranged 20-65 with mean $36.52 \pm 11.81$

Table (2): ASA distribution of the study group $(\mathrm{N}=70)$.

\begin{tabular}{|l|c|c|}
\hline \multicolumn{1}{|c|}{ ASA } & No. & \% \\
\hline III & 56 & $80.0 \%$ \\
\hline IV & 14 & $20.0 \%$ \\
\hline Total & 70 & $100.0 \%$ \\
\hline
\end{tabular}

This table demonstrates that the ASA III $(80 \%)$ and ASA IV (20\%) of ASA.

Table (3): Causes distribution of the study group $(\mathrm{N}=70)$.

\begin{tabular}{|l|c|c|}
\hline \multicolumn{1}{|c|}{ Causes } & No. & \% \\
\hline Post-surgical & 24 & $34.29 \%$ \\
\hline Sepsis & 20 & $28.57 \%$ \\
\hline Obstetrics & 12 & $17.14 \%$ \\
\hline Poly trauma & 9 & $12.86 \%$ \\
\hline Malignancies & 5 & $7.14 \%$ \\
\hline Total & 70 & $100.00 \%$ \\
\hline
\end{tabular}

This table illustrates that the Obstetrics (17.14\%), Sepsis (28.57\%), Poly trauma (12.86\%), Post-surgical (34.29\%) and Malignancies (7.14\%) of causes.

Table (4):Fibrinogen/CRP ratio distribution of the study group $(\mathrm{N}=70)$.

\begin{tabular}{|l|c|c|}
\hline Fibrinogen / CRP ratio & No. & \% \\
\hline Positive & 39 & $55.7 \%$ \\
\hline Negative & 31 & $44.3 \%$ \\
\hline Total & 70 & $100.0 \%$ \\
\hline
\end{tabular}

This table reveals that the positive (55.7\%) and negative (44.3\%) of Fibrinogen/CRP ratio.

Table (5): FDPs+ D dimer distribution of the study group $(\mathrm{N}=70)$.

\begin{tabular}{|l|c|c|}
\hline \multicolumn{1}{|c|}{ FDPs+D dimer } & No. & \% \\
\hline Positive & 64 & $91.4 \%$ \\
\hline Negative & 6 & $8.6 \%$ \\
\hline Total & 70 & $100.0 \%$ \\
\hline
\end{tabular}

This table displays that the positive (91.4\%) and negative (8.6\%) of FDPs+D dimer.

Table (6):Comparison between results Fibrinogen /

CRP ratio and FDPs + D dimer $(\mathrm{N}=70)$.

\begin{tabular}{|l|c|c|c|}
\hline \multicolumn{1}{|c|}{ Parameters } & No. & Sensitivity & p-value \\
\hline Fibrinogen / CRP ratio & 39 & $55.7 \%$ & \multirow{2}{*}{$<0.001^{* *}(\mathrm{HS})$} \\
\hline FDPs+D dimer & 64 & $91.4 \%$ & \\
\hline
\end{tabular}

This table indicates highly statistically significant difference between results Fibrinogen / CRP ratio and FDPs+D dimer.

\section{DISCUSSION}

Disseminated intravascular coagulopathy is a serious complication with different etiologies which requires early detection for early intervention as early intervention in DIC improves prognosis ${ }^{(\mathbf{1})}$.

There are many laboratory measures and scores for diagnosis of DIC like fibrinogen, PT, PTT, platelets count, FDPs and D-dimer,in addition to its clinical findings; but allthese laboratory measures alone lack sensitivity ${ }^{(\mathbf{8})}$.

A new measure Fibrinogen/CRP for diagnosis of DIC was adopted by Kim et $\boldsymbol{a l}^{\left({ }^{(5)}\right.}$ and according to this study low fibrinogen/CRP was highly correlated with high DIC score.Plasma fibrinogen level was well correlated with CRP level, as fibrinogen/CRP ratio could eliminate the 
increased fraction of fibrinogen that is caused by acute protein synthesis and this ratio is well correlated with serum FDPs so fibrinogen /CRP ratio can be used as a sensitive marker of coagulation factor consumption for overt DIC still,this ratio has many limitations.

First fibrinogen level and CRP were high enough in patients with liver disease and hematological diseases which can overestimate DIC, also CRP kinetics can't be compared with fibrinogen kinetics during acute synthesis of these proteins as CRP rises significantly within 12 hours after an acute inflammatory insult reaching maximum levels in 2-5 days ${ }^{(9)}$ and it is also known that after injury plasma, fibrinogen rises gradually and is sustained at the postinjury level ${ }^{(10)}$.

Even though both proteins are increased during the acute phase, it can't be confirmed that, the increment amount of both these parameters are due to acute inflammatory response, therefore fibrinogen/CRP ratio may not reflect accurate consumption of fibrinogen ${ }^{(11)}$.

In our study, a comparison was done between fibrinogen/CRP ratio and 2 classical laboratory parameters which are FDPs + D-dimer, in order to find out a sensitive laboratory tool for the diagnosis of DIC.

In the present study, a total sample of 70 patients were gathered from Ain shams surgical ICU (postoperative surgical ICU and OBS/GYN ICU) suffering from acute DIC according to ISTH score which is considered most sensitive score in diagnosing overt DIC.

Two samples were withdrawn from each patient and divided into 2 groups.

The first group is analyzed for fibrinogen/CRP ratio and second group for Ddimer and FDPs.

The patients were analyzed according to their age, etiology and ASA score.

According to the study, the most age group suffering from DIC were around 20-30 and 30-40 years, this can be explained that this age group (child bearing period) which shows the most common cause of DIC are obstetric, postsurgical and malignancies,40-50 years due to malignancies and postoperative.

Also our study showed the most common etiologies of DIC which were postsurgical 34.29\%, sepsis $28.57 \%$, obstetrics $17.14 \%$, polytrauma $12.86 \%$ and malignancies $7.14 \%$.

After samples were taken, fibrinogen/CRP ratio was done for first group the ratio <104 was considered to be positive and >104 is negative ratio.

After tabulating and analyzing the results sensitivity of fibrinogen/CRP ratio $=55 \%$ according to our study meanwhile according to Kim $\boldsymbol{e t}$ al. ${ }^{(\mathbf{5})}$ ratio is $60 \%$

Samples of the second group was done as FDPs $>40 \mathrm{mg} / \mathrm{ml}$ is considered positive value, and D-dimer value $>300 \mathrm{mcg} /$ lit was considered a positive value.

After tabulating and analyzing the results of the second group sensitivity $=90 \%$ also like the

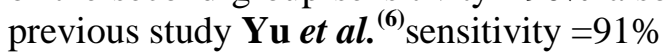

Results of our study agree with those of $\mathbf{Y u}$ et $\boldsymbol{a l}{ }^{(\boldsymbol{6})}$, first due to similar number of patients (82 patients), and the same study duration., as well as the resemblance of inclusion and exclusion criteria.

\section{CONCLUSION}

The D-dimer and FDP tests offered the best test panel in the diagnosis of DIC especially in overt DIC due to their high sensitivity.

\section{REFERENCES}

1. Costello R, Nehring $S$, Coover $C$ andBayot $M$ (2017): Disseminated intravascular coagulation (DIC). StatPearls, 154(1):26-34.

2. Taylor FB Jr, Toh CH, Hoots WK, Wada H and Levi M (2001): Towards definition, clinical and laboratory criteria, and a scoring system for disseminated intravascular coagulation. ThrombHaemost., 86(5):1327-30.

3. Semeraro N, Ammollo CT, Semeraro F and Colucci M (2010): Sepsis-associated disseminated intravascular coagulation and thromboembolic disease. Mediterranean journal of hematology and infectious diseases, 2(3): 112-121.

4. Thachil J (2016): Disseminated Intravascular Coagulation: A Practical Approach. Anesthesiology: The Journal of the American Society of Anesthesiologists, 125(1):230-6.

5. Kim HK, Lee DS, Kang SH, Kim JQ, Park S and Cho HI (2007): Utility of the fibrinogen/Creactive protein ratio for the diagnosis of disseminated intravascular coagulation. Actahaematologica,117(1):34-9. 
6. Yu M, Nardella A andPechet $L$ (2000): Screening tests of disseminated intravascular coagulation: guidelines for rapid and specific laboratory diagnosis. Critical care medicine, 28(6):1777-80.

7. Windsperger $K$ andLehner $R$ (2013): The fibrinogen/CRP ratio as a new parameter for the diagnosis of disseminated intravascular coagulation in patients with HELLP syndrome and as a predictive factor for neonatal outcome. Am JObstetGynecol.,208:118.el-7.

8. Boral BM, Williams DJ, Boral LI (2016): Disseminated intravascular coagulation. American journal of clinical pathology, 146(6):670-80.

9. Jain S, Gautam V andNaseem S (2011): Acute-phase proteins: As diagnostic tool. Journal of Pharmacy and Bioallied Sciences, 3(1):118.
10. Schlimp CJ, Ponschab M, Voelckel W, Treichl B, Maegele M, Schöchl H (2016): Fibrinogen levels in trauma patients during the first seven days after fibrinogen concentrate therapy: a retrospective study. Scandinavian journal of trauma, resuscitation and emergency medicine, 24(1):29.

11. Chandy S, Joseph K, Sankaranarayanan A, Issac A, Babu G, Wilson B and Joseph J (2017): Evaluation of C-reactive protein and fibrinogen in patients with chronic and aggressive periodontitis: a clinico-biochemical study. Journal of clinical and diagnostic research: JCDR., 11(3):ZC41. 